\title{
Study on Fish and Vegetable Co-existing System Based on Wireless Sensor Network
}

\author{
Yueling Zhao ${ }^{1, a^{*}}$, Zichao Ma ${ }^{1, b}$, Haiyan $\operatorname{Han}^{2, \mathrm{c}}$ and Guifen Chen ${ }^{1, \mathrm{~d}}$ \\ ${ }^{1}$ Jilin Agricultural University, Changchun, 130118, China; \\ ${ }^{2}$ Changchun University, Changchun, 130000, China \\ azyueling@163.com, b275714495@qq.com, ‘453876175@qq.com, d1045093555@qq.com
}

\begin{abstract}
Keywords: Fish and vegetable; Co-existing system; Wireless sensor network; ZigBee
\end{abstract}
\begin{abstract}
With the rapid development of the national economy, low carbon economy has become a new type advocated economy in modern agriculture. This paper presents a research method of water quality monitoring system based on wireless sensor network. It is the basis to save energy and to reduce emission in the agricultural development by using the principle and method of the sensor, using CPU-ARM chip, LCD touch screen, temperature sensor, $\mathrm{pH}$ sensor, liquid level sensor, MP3 module, light, and feed device. It can be realized to real time detection of environmental temperature and $\mathrm{pH}$ value, automatic feeding the fish, fish dishes on the water circulation system of remote control function etc.
\end{abstract}

\section{Introduction}

With the continuous development of science and technology, wireless sensor network has step into the people's attention. In the typical application of wireless sensor networks, the application of the initial military system gradually infiltrated all aspects of life, such as environmental monitoring, health management, etc. Generally a node's data is only a few bytes, the amount of data and the number of nodes become more less [1-2]. These sensor networks generally use ZigBee protocol, which is mainly used to collect temperature, humidity and other sensor data in the environment. There are a large amount of wireless sensor network recovery data, these sensor networks are usually based on embedded devices, using WIFI technology, data recovery usually use FTP or UDP protocol.

Wireless sensor network is one of the internet things. It is an important carrier to change the agricultural production mode. It is important type to promote the sustainable development of agriculture and to utilize the rural resources efficiently in China. As a wireless information acquisition and processing technology in the field of agricultural production. It is concerned widely [3-4]. In view of the deficiency of artificial control, the author applies the wireless sensor network technology in the course of the course of the fish and vegetable co-existing system, and designs the system of food and symbiotic system based on wireless sensor network. This system makes full use of ZigBee communication technology, the temperature and humidity of fish and vegetable coexisting system are collected, so as to ensure the aquaponics environment always maintain the best state of aquaculture to provide reference to the development of intelligence.

\section{Overall Structure of the System}

The fish food symbiotic system are carefully selected and applied to a variety of sensors to detect fish and vegetable co-existing system interior various environment, such as illumination, air temperature and humidity, and the information such as the $\mathrm{PH}$ of the water, using the Android smartphone remote monitoring system, ensure the safety of fish food symbiotic system efficiently. Intelligent monitoring system adopts distributed structure. Each part has a combination or independent form. In general, the vegetable co-existing monitoring system can be divided into wireless sensor network, embedded gateway and smartphone monitoring terminal three parts. The fish vegetable co-existing system diagram is as shown in Fig. 1. 


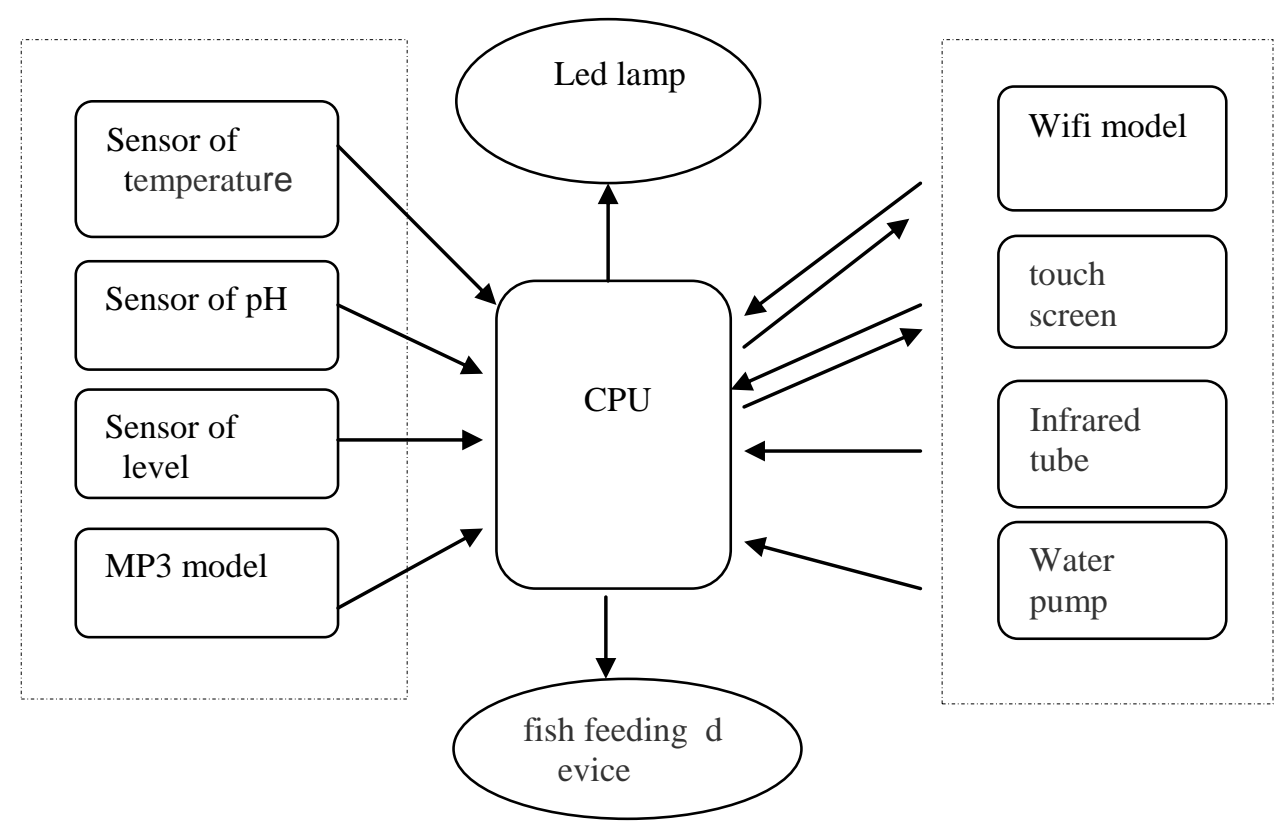

Figure 1. Overall structure of fish vegetable co-existing system

\section{System Module Implementation}

Wireless Sensor Network . The fish and vegetable co-existing system using sensors collect plant growth of vegetables in the area of information. Sensor network hardware platform is controlled by various kinds of sensor node, the coordinator, node and the execution parts. The sensor nodes and between nodes and the coordinator controlled by ZigBee module communication. Perform component and control via a serial port communication between nodes [5]. Wireless sensor network (WSN) bear the fish and vegetable co-existing monitoring area of all kinds of sensor data acquisition and execute device control work ${ }^{[6]}$. Sensor nodes equipped with temperature and humidity sensor, humidity sensors, light sensors and other sensor nodes through the ZigBee module ad-hoc network, collaboration between each node, in addition to the information data collection and processing, also for other nodes forwarded data forwarding, etc., each node to complete the monitoring task ${ }^{[7-9]}$. Data collected in accordance with the upload cycle of software to send to the coordinator, and then through the serial bus real-time data transmission to the embedded gateway.

The fish and vegetable co-existing system of wireless sensor network node adopts modular design, can be divided into core board, sensor module, control module, main board and so on four parts. Core board master control chip USES the ZigBee wireless communication chip CC2430, responsible for driving sensor and data receiving and sending. Sensor module including temperature and humidity sensor, humidity sensor, light sensor, responsible for indoor environment information collection, perceived environmental changes. Realize the control of switch quantity control module, mainly composed of four relay and control circuit, control execution unit to adjust the environment changes. The perform parts included heater, humidifier, submersible pump and LED plant light, is responsible for regulating the fish food factory indoor environment.

The Gateway of Embedded. Embedded gateway is mainly composed of ARM embedded system and its external expansion device camera, and other components of the wireless network card, wireless router, embedded gateway and wireless sensor network communication by the coordinator. The gateway of the system is embedded responsible for data reception, data processing and coordinate processing, and other functions. Processor is mainly done for the coordinator to send to the information such as the soil humidity, air temperature and humidity, light make a comprehensive analysis, at the same time according to the internal software written perform 
component of automatic control in wireless sensor network (WSN), at the same time the LCD screen will display the air temperature and humidity real-time information, light, water and so on. The user can through the intelligent mobile terminal check fish and plant vegetables growth.

The Phone Terminal. Smartphone monitoring terminal is in charge of the sensor data visualization query and remote control command sent, and for human-machine interface for users of related parameters Settings. Users can through the smart phone access to the data collected by the wireless sensor network (WSN), and through the balance of the terminal setting system parameters. This way of real-time query and feedback to avoid a large number of sensing information sent at the same time caused by network congestion problem, strong real-time performance, improve query fish and vegetable co-existing system within the efficiency of ecological environmental data. In order to be able to monitor anytime and anywhere, the user can through the intelligent mobile terminal in vegetable factory fully monitoring state of real-time data monitoring area. Service request module is responsible for the data query request instructions and configuration on the package sent to the embedded gateway. Data reception processing module will receive the packet decoding, analysis, and carry on corresponding processing.

\section{The Analysis of System Function}

Function Introduction of the System. The device has a manual operation mode and PC operation mode: the user can choose according to their mode control CPU want function, etc. The principle is the fish tank water and water bed realize recycling, fish excrete waste can be sampled with water pump to vegetables, the vegetables to absorb, to achieve the effect of water purification, purification of water to flow back into the fish tank. The cycle of fish dishes within the symbiotic effect.

The system unit is divided into a main menu and six sub menus. The first is the temperature sub menu. When pressed by hand when the temperature of the ICONS on the screen, program into the temperature for fish tank water temperature in the sub menu. When the temperature higher or lower than a certain range, the buzzer alarm happens, the screen will display the alarm information. Remind users to take certain measures. The second is the PH submenu. When hand press the PH icon on the screen, the program into the $\mathrm{PH}$ in the sub menu for fish tank water $\mathrm{PH}$. When $\mathrm{PH}$ value higher or lower than a certain range, the buzzer alarm happens, the screen will display the alarm information. The third is the infrared control of tube light mode, lights flashing, with fish swimming The fourth is to feed the fish and music mode submenu. In the sub menu, there are two buttons, one for music pattern, a model for feed on fish. The fifth is remote control mode. It can be connected to PC through the wifi data remote access to temperature, $\mathrm{pH}$ value, control, and so on. The sixth is liquid level accessing mode. It included the balance of water level, manual pumping mode, etc.

\section{Conclusions}

This article mainly studies the embedded microcontroller processor articulated data acquisition module and wireless communication module to complete the fish and vegetable co-existing information remote monitoring. Its first converts depended the $12 \mathrm{v}$ power supply circuit voltage $5 \mathrm{v}$ voltage, at the same time using the running speed, low power consumption M20 reset circuit of GPRS module hardware circuit was improved; In view of the existing ater information wireless communication more slowly, good software and hardware cannot match, and the hardware design of the defects of difficult, through correction GPRS management software and GPRS communication software program flow scheme, thus finally makes up for the traditional remote monitoring system of fish water high investment, low extensibility, wiring, the shortcomings of low intelligence. The test showed that system reliable operation, provides to realize intelligent precise management technology research model, can be used for reference to promote the development of the fish and vegetable co-existing monitoring control technology. It is great significance of future. 


\section{Acknowledgements}

Funds for the research was provided by scientific research fund of Jilin Agricultural University (No.201620), Jilin province university undergraduate science and technology innovation fund. National university undergraduate science and technology innovation fund (No.201510193040).spark plan Ministry of science and technology (2015GA660004).

\section{References}

[1] XiaoweiLuo, William J. O'Brien, Christine L. Julien .Comparative evaluation of Received Signal-Strength Index (RSSI) based indoor localization techniques for construction job sites[J].Advanced Engineering Informatics,2011,25 : 355-363.(In Chinese).

[2] Jungang Zheng, Chengdong Wu, HaoChu, etc. An Improved RSSI Measurement in Wireless Sensor Networks [J].Procedia Engineering 2011, 15: 876-880. (In Chinese).

[3] Xinwei Wang, Shaoping Yuan, Rainer Laur,etc. Dynamic localization based on spatial reasoningwith RSSI in wireless sensor networks for transport logistics[J].Sensors and Actuators,2011,171:421-428. (In Chinese)

[4] Wang Fulu, jung dragon, Zhang Xihai. The greenhouse environment monitoring system based on wireless sensor network research [J]. Journal of northeast agricultural university, 2011, 42 (2): 59-64. (In Chinese).

[5] chang-chunbao, rui-zhenshi, yu-quan ma, etc based on ZigBee technology of measurement and control system of agricultural facilities design [J]. Journal of agricultural engineering, 2007, 23 (8): 160-164. (In Chinese).

[6] Hongyang Chen, Kaoru Sezaki, Ping Deng, et al. An improved DV-Hop Localization Algorithmfor Wireless Sensor Network [J]. In: Proceedings of the 3rd IEEE Conference on Industrial Electronic and applications, 2008: 1557-1561. (In Chinese).

[7] Sun Daozong, wei-xingwang, Jiang Sheng. Based on soil moisture measurement and modeling of double sensors data fusion [J]. Journal of agricultural engineering, 2012, 28 (15): 60-64.

[8] Niculescu D, Nath B. DV based positioning in ad hoc networks [J]. Journal of Telecommunication Systems, 2003, 22 (1/4):267-280.

[9] Wen-chuanguo, Cheng Hanjierui-ming li, etc. The greenhouse environmental information monitoring system based on wireless sensor network [J]. Journal of agricultural machinery, 2010, 9 (7): 181-185. (In Chinese). 